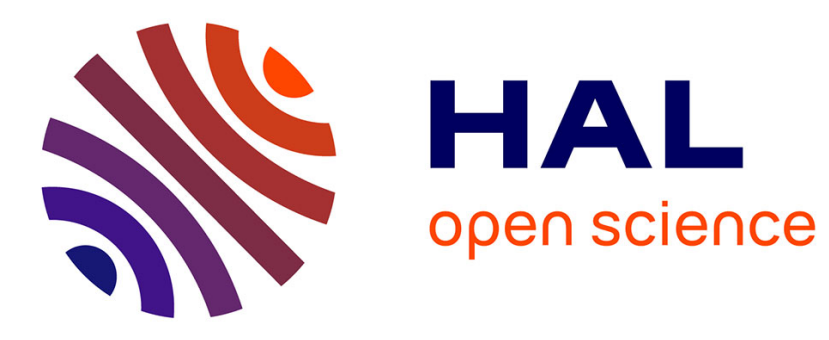

\title{
Les syndicalismes arabes au prisme de l'autoritarisme et du corporatisme
}

\author{
Eric Gobe
}

\section{To cite this version:}

Eric Gobe. Les syndicalismes arabes au prisme de l'autoritarisme et du corporatisme. de Olivier Dabène, Vincent Geisser, Gilles Massardier. Autoritarismes démocratiques et démocraties autoritaires: Convergences Nord-Sud, La Découverte, p. 267-284, 2008, Recherches. halshs-00398569

\section{HAL Id: halshs-00398569 \\ https://shs.hal.science/halshs-00398569}

Submitted on 24 Jun 2009

HAL is a multi-disciplinary open access archive for the deposit and dissemination of scientific research documents, whether they are published or not. The documents may come from teaching and research institutions in France or abroad, or from public or private research centers.
L'archive ouverte pluridisciplinaire $\mathbf{H A L}$, est destinée au dépôt et à la diffusion de documents scientifiques de niveau recherche, publiés ou non, émanant des établissements d'enseignement et de recherche français ou étrangers, des laboratoires publics ou privés. 


\section{Les syndicalismes « arabes » au prisme de l'autoritarisme et du corporatisme}

\section{ÉRIC GOBE*}

Le propos est ici d'analyser les rapports entre le pouvoir politique et les organisations syndicales dans le monde arabe. Par ce biais, j'étudierai une des modalités par lesquelles certains États autoritaires assurent leur hégémonie sur la société et se reproduisent sur des modes sans cesse renouvelés.

Je m'intéresserai plus particulièrement à trois pays arabes : l'Égypte, la Tunisie, et l'Algérie ${ }^{1}$. Dans leur histoire récente et durant une période plus ou moins longue, ces États ont connu une forme d'organisation politique qui les rattache à ce que certains politologues ont dénommé l'autoritarisme populiste. Ce concept recouvre un type de régime politique dans lequel les gouvernants, dotés d'une idéologie se réclamant le plus souvent du socialisme et mythifiant le peuple présenté comme un corps soudé, mettent en œuvre « une stratégie de développement qui cherche à atténuer les conséquences négatives de la croissance pour certains groupes sociaux (les ouvriers, les paysans, la petite bourgeoisie urbaine) aux dépens d'autres, plus particulièrement les capitalistes et les propriétaires fonciers »[Heydemann, 1999, p. 5]. Dans cette perspective, les auteurs qui ont tenté de décrire les traits de l'autoritarisme populiste mettent l'accent sur «les formes de mobilisation, de formation de coalitions et d'institutionnalisation destinées à encadrer les catégories sociales “populaires" à l'intérieur d'arrangements politiques et économiques » [Ibid.]. Ce faisant, ils se sont largement inspirés des définitions du populisme données par les politologues ayant travaillé sur l'Amérique. Le populisme est ainsi analysé comme une tentative d'intégration par le haut «des secteurs sociaux les défavorisés autour d'un projet idéologique aux contours flous » [Dabène, 2007, p. 67].

Cette stratégie de développement articulée à une politique d'industrialisation par substitution aux importations s'est accompagnée d'une forte étatisation de l'économie. Cette expérience a profondément marqué la trajectoire des États et les relations qu'ils entretiennent avec leur société.

Au sortir de la lutte pour l'indépendance en Tunisie et en Algérie et à peu près à la même période en Égypte, les gouvernants ont construit des régimes politiques populistes et autoritaires s'appuyant sur ce que Philippe Schmitter [1974] appelle le « corporatisme d'État » ou corporatisme autoritaire.

Le principal objectif du corporatisme autoritaire, en Tunisie comme en Égypte et

* Éric Gobe est chercheur à l'Institut de recherche sur le monde arabe et musulman (IREMAM/CNRS)

${ }^{1}$ Les États du Golfe ne sont pas évoqués dans cet article. Mais leur trajectoire historique et leur structure sociale expliquent la quasi-absence de mouvements syndicaux, à l'exception du Yémen et du Koweït. Les syndicats sont encore interdits en Arabie Saoudite, au Qatar, à Bahreïn et dans les Émirats Arabes Unis (les pouvoirs publics y ont rédigé en 2004 un projet de création d'une Fédération nationale du travail). Oman a récemment autorisé les ouvriers à mettre en place des «comités » destinés à les représenter auprès des autorités. Voir à ce sujet le site de la Confédération internationale des syndicats libres consulté le 20 août 2005. 
en Algérie, était d'encadrer des groupes sociaux populaires dans des structures verticales de mobilisation au profit d'un projet national de développement se réclamant du socialisme.

Les diverses idéologies corporatistes qui sous-tendent la mise en place des différentes formes de corporatisme autoritaire développent une vision organique de la société qui refuse le conflit ainsi que l'idée de lutte des classes. Elles présentent la société comme un corps composé de groupes fonctionnellement complémentaires qui s'insèrent dans des organisations hiérarchisées, disciplinées, non concurrentielles représentant les intérêts de leurs membres vis-à-vis de l'État. Les gouvernants peuvent donner à ces organisations des fonctions quasi-étatiques dans leur catégorie respective et coopter leurs dirigeants dans les institutions gouvernementales. Dans ce cadre, les négociations portant sur l'allocation des ressources se déroulent non pas entre les différents groupements, mais entre ceux-ci et les gouvernants qui constituent à la fois le « cerveau » et l'arbitre des divers intérêts [Richards, Waterbury, 1990, p. 337]. Dans la mesure où le corporatisme autoritaire tend à organiser la société en groupes fonctionnels verticaux ainsi qu'à prévenir l'expression d'intérêts pluralistes et concurrentiels, il constitue un instrument de contrôle et d'encadrement des divers groupes sociaux dans leur accès aux ressources matérielles.

$\mathrm{Au}$ cours de ces vingt dernières années, des politologues comme Nazih Ayubi [1995], Robert Bianchi [1989], Ninette Fahmy [2002], Emma C. Murphy [1999] Alan Richard et John Waterbury [1990] ont repris et enrichi l'approche de Philippe Schmitter [1974] en l'inscrivant dans la dynamique historique des pays arabes. Nazih Ayubi a fait du corporatisme l'une des clés d'analyse des systèmes politiques dans le monde arabe. Il opère une distinction entre un «corporatisme stato-centré » et un «corporatisme socio-centré ». Ce dernier émergerait dans des États en voie de modernisation (l'Empire ottoman des XVIII ${ }^{\mathrm{e}}$ et $\mathrm{XIX}^{\mathrm{e}}$ siècles et les pays de la péninsule Arabique au $\mathrm{XX}^{\mathrm{e}}$ siècle) et constituerait un effort d'intégration des groupes précapitalistes (tribaux, ethniques ou religieux) dans un processus de transformation capitaliste inachevé. Quant au corporatisme stato-centré, il se rapporterait dans le Monde arabe aux «pays d'industrialisation tardive » qui ont développé une forme de capitalisme encadrée par l'État, imprégnée d'idéologie nationaliste et soutenue par une «techno-bureaucratie » en voie de constitution. Dans le cadre de ce corporatisme, les gouvernants se sont appuyés sur certaines organisations syndicales pour éliminer les groupes contestant leur légitimité et pour favoriser la conclusion d'une alliance entre les différentes catégories sociales populaires dont aucune ne pouvait exercer un pouvoir hégémonique sur l'ensemble de la société. Selon Ayubi [1995, p. 191-192], le «corporatisme stato-centré » articulé à l'autoritarisme populiste trouverait sa réalisation la plus achevée dans l'Égypte nassérienne et, dans une moindre mesure, dans la Tunisie, l'Algérie, la Syrie et l'Irak des années 1960-1970.

Aujourd'hui, il n'est plus possible de définir ces régimes politiques de populistes autoritaires même si, par un effet d'hystérésis, certains de leurs éléments perdurent bien qu'aient disparu les facteurs qui avaient présidé à leur apparition.

Ils se sont progressivement transformés dans les années 1970 concentrant de plus en plus de traits de «l'autoritarisme bureaucratique ». Autrement dit, ils s'assignent une «fin modernisatrice» tout en cherchant à esquiver «le danger de subversion sociale impliquée impliqué par les stratégies populistes antérieurs » [Badie, Hermet, 
2001, p. 195]. Dans ce nouveau contexte, les gouvernants s'efforcent de modeler le contexte économique et social d'une manière plus favorable à l'expertise technocratique [Linz, 2000] et, au bout du compte, à partir des années 1980, à l'expansion du secteur de l'entreprise privée. Le corporatisme lui-même évolue et tend alors à remplir une fonction de contrôle qui « se réalise en partie par la répression [...]. Mais il se combine aussi avec la prévention et l'élimination des conditions qui permettraient la réapparition de modes d'organisation et de formulation de revendications » [Guillermo O’Donnell cité dans Droz-Vincent, $\mathrm{p}$ 204].

Reprenant la terminologie de Robert Bianchi [1989], on considère que ces régimes s'efforcent de «corporatiser» les organisations syndicales et professionnelles. Cette «corporatisation» (corporatization) $)^{2}$ participe d'une mise sous tutelle de la société.

Mais certains secteurs socioprofessionnels ont été plus concernés que d'autres. Sans trop caricaturer, on peut dire que les « syndicats ouvriers » ont été beaucoup plus rapidement et profondément corporatisés que les institutions représentant les «professions libérales». En effet, les «syndicats ouvriers», conçus comme des instruments de «médiation » avec le monde salarié (surtout du secteur public), ont été vus par les gouvernants des États autoritaires comme des institutions chargées de désamorcer les revendications et les protestations sociales [Richards, Waterbury, 1990, p. 340-341].

\section{Régimes populistes autoritaires et corporatisation du mouvement syndical}

L'appellation de syndicat ouvrier ne doit pas faire illusion : les ouvriers n'y étant pas toujours majoritaires, il serait préférable de parler de syndicats de salariés regroupant «cols bleus » et «cols blancs» [Richards, Waterbury, 1990, p. 340]. En effet, le syndicalisme représente principalement le salariat public et manifeste ainsi «sa dépendance de l'État national social qui est deux fois distributeur de revenus, par le salariat public et par les avantages sociaux $»^{3}$. Que la politique économique proclamée ait plus ou moins fortement étatisé l'économie, l'effet a partout été dans les années 1960 d'élargir le secteur public, et principalement « le salariat tertiaire public, à l'intérieur même des entreprises » [Gallissot, 1987, p. 379]. Ne fonctionnant qu'à titre second comme un mouvement ouvrier, le syndicalisme est, pour reprendre l'expression de René Gallissot, défini par sa «fonction d'encadrement», les permanents syndicaux étant principalement «des employés bureaucrates parlant au nom de la classe ouvrière ». Dans ce cadre, les cadres syndicaux et les directions des sociétés du secteur public ont toute une série d'intérêts sociopolitiques en commun : il convient que les entreprises d'État connaissent des performances économiques raisonnables; il s'agit de négocier au mieux avec les ministères de tutelle l'obtention de ressources pour faire tourner les entreprises et distribuer des avantages matériels. Les directions du syndicat et des sociétés publiques ont un égal intérêt à garder la base du syndicat sous contrôle: toute opposition radicale susceptible d'exploiter le

2 J'aurai pu traduire ce concept par «encadrement», mais cet anglicisme a l'avantage de décrire un processus dans lequel les organisations syndicales et professionnelles sont conçues comme des instruments d'encadrement et de mobilisation de la société.

3 René Gallissot, préface à l'ouvrage d'Amar Benamrouche [2000, p. 8]. 
mécontentement des travailleurs contre le régime doit être supprimée. Les cadres syndicaux sont ainsi clientélisés et se préoccupent davantage de faire fructifier les gratifications matérielles et symboliques inhérentes à leur position que de défendre les intérêts de la base. Leur conduite est davantage dictée par leurs rapports avec l'État que par les revendications sociales des adhérents au syndicat.

\section{Égypte : le régime nassérien, inspirateur de l'autoritarisme populiste dans le monde arabe}

L'Égypte de Nasser constitue, dans la région, le premier État à avoir mis en place une formule politique relevant de l'autoritarisme populiste et corporatiste. Pourtant, la création d'une organisation « ouvrière » unique, non concurrentielle et hiérarchisée, date seulement de 1957, soit cinq ans après la prise du pouvoir par les Officiers libres. Certes, le régime nassérien comptait bien s'appuyer sur le salariat en expansion pour consolider son pouvoir, mais dans le même temps, il ne voulait pas se trouver piégé par une organisation syndicale trop puissante. De leur côté, certains dirigeants syndicaux souhaitaient unifier le «mouvement ouvrier» égyptien au sein d'une organisation unique. Nasser n'accepte la création d'une centrale unique qu'en échange $\mathrm{du}$ droit pour le gouvernement de nommer les dirigeants de la nouvelle fédération [Posusney, 1997, p. 63].

C'est également à la fin des années 1950 que se mettent en place les éléments du compromis social populiste nassérien : le régime met en œuvre une politique sociale généreuse que les dirigeants de la centrale syndicale égyptienne sont chargés d'expliquer à la base. Cette politique est d'autant mieux acceptée par les catégories populaires salariées que dans l'ensemble, elles bénéficient de meilleures conditions de travail et d'une réévaluation des salaires. Toute une série de mesures prises dans les années 1960 est emblématique de cette politique visant à s'assurer le soutien des classes populaires tout en évitant l'émergence de mouvements sociaux potentiellement déstabilisateur. En 1959, est promulgué le code unifié du travail qui prévoit, entre autres, la réduction de la journée de travail à huit heures. Les «législations socialistes » de 1961 qui nationalisent les grandes entreprises égyptiennes débouchent sur un élargissement considérable du secteur public. La loi 114 de 1961 prévoit la représentation des salariés au conseil d'administration des entreprises publiques et celle de 1963 en augmente le nombre. Ce dispositif législatif tente d'évacuer le conflit en prohibant certains comportements comme «l'incitation à la lutte des classes » et l'organisation d'arrêts de travail (le texte autorise les fonctionnaires à adhérer à la Confédération). La nouvelle constitution égyptienne promulguée en 1965 parachève le projet corporatiste nassérien en introduisant une « représentation fonctionnelle » des catégories sociales dans toutes les instances représentatives, à l'Assemblée du peuple, dans les conseils locaux et au sein du parti : «Trois grandes catégories sont définies : les “'ouvriers"' les “paysans", et les ' autres catégories', lesquelles incluent, outre les commerçants et les propriétaires, l'ensemble des catégories intermédiaires diplômées » [Longuenesse, 2007, p. 130].

Sous les présidences de Sadate et de Moubarak, ce compromis social populiste s'est retrouvé progressivement écorné, alors que la mise sous tutelle de la centrale syndicale ne s'est jamais démentie. Pour reprendre la terminologie de Ninette Fahmy, 
l'Égypte serait passée d'un «corporatisme populiste», inscrit dans une logique redistributive large, à un « corporatisme co-intégrationniste » assimilé à un système de contrôle de l'État sur les différents groupes d'intérêt. Cooptant les dirigeants de ces syndicats et groupes professionnels, l'État leur distribue par le biais de réseaux clientélistes des avantages matériels et symboliques en échange de leur soutien. Dans le cadre de cette formule corporatiste, les gouvernants essaient d'affaiblir et de réduire l'influence politique des différents groupes socioprofessionnels à travers la répression, les interventions dans le processus de sélection des candidats aux élections syndicales, l'instrument législatif ainsi que la distribution de privilèges à ses soutiens potentiels [Fahmy, 2002, p. 105-106].

Pendant toute la présidence de Sadate (1970-1981) et sous une partie de celle de Moubarak, le président de la Confédération générale des syndicats des travailleurs d'Égypte (CGSTE) est également ministre du Travail, ce qui contribue à accentuer le caractère « étatique » du corporatisme syndical égyptien.

À partir de 1976, les candidats à des fonctions électives ne sont plus obligés d'être membres du parti gouvernemental, mais les différents gouvernements s'invitent sans cesse dans les élections aux comités syndicaux pour limiter au maximum la présence de membres de l'opposition. Le ministère du Travail a toujours supervisé les procédures électorales aux différents niveaux de la Confédération et intervient en amont du scrutin en décourageant, parfois par la menace, des candidatures non désirées [Ibrahim, 1998, p. 194-195].

Les techniques visant à écarter les opposants ou les candidats indépendants sans lien avec le parti au pouvoir, le Parti national démocrate (PND), sont innombrables et le verrouillage de l'accès aux niveaux supérieurs de la hiérarchie de la Confédération est encore plus efficace [Kienle, 2000, p. 81-84]. Les dirigeants de la CGSTE qui siègent sous les couleurs du PND au parlement et dans les conseils municipaux [Clément, 1998, p. 44] n'ont jamais initié ou soutenu un mouvement de grève au niveau des entreprises. Cependant, pour tenter de préserver un semblant de crédibilité auprès de ses membres et, par conséquent, avoir une certaine utilité pour les gouvernants, la direction de la Confédération s'est posée, dans les années 1970 et 1980, comme le défenseur de causes «nationales » ayant un certain écho auprès de la base - plus particulièrement la défense du secteur public et le refus de son éventuelle privatisation [Posusney, 1992]. Avec la mise en œuvre, dans les années 1990, d'une politique de privatisation, la coupure entre la direction syndicale et la base va s'approfondir.

\section{Algérie : la centrale syndicale gardienne de la « révolution socialiste»}

L'Algérie apparaît au Maghreb comme le parangon de l'économie étatisée et du populisme. La centrale syndicale algérienne, l'Union générale des travailleurs algériens (UGTA) a été créée en 1956, sur injonction du Front de libération nationale (FLN), par des syndicalistes algériens de la Confédération générale des travailleurs (CGT) et de la Confédération française des travailleurs chrétiens désireux de s'inscrire dans la lutte pour l'indépendance de l'Algérie. En fait, l'UGTA «n'est pas [pour reprendre la terminologie marxiste du sociologue algérien Saïd Chikhi, 1982, p. 65] une conquête de la classe ouvrière mais le produit de l'État parti pour les ouvriers ». 
Dès son premier congrès de 1963, le président de la République, Ahmed Ben Bella, envoie des «faux délégués manipulés par le FLN », le parti unique, pour évincer la «direction représentative » [Ahdjouj, 1992, p. 105]. Si le deuxième congrès, en 1965, voit émerger une équipe dirigeante autonome, son refus d'adopter une déclaration de soutien au pouvoir issu du coup d'État de Boumediene aboutit à son éviction et à l'installation d'une direction entièrement subordonnée au FLN et au nouveau gouvernement [Benamrouche, 1995, p. 47-48]. L'UGTA devient au début de la décennie 1970 un appareil chargé de conscientiser, d'encadrer et de mobiliser les salariés au service de la «révolution socialiste et ses conquêtes » selon la logique du corporatisme autoritaire : elle inscrit dans ses statuts lors du IV ${ }^{\mathrm{e}}$ congrès de 1973 qu'elle est désormais une « organisation de masse du parti d'avant-garde FLN $»^{4}$.

Toutefois, mise sous tutelle, la centrale syndicale algérienne se retrouve incapable de désamorcer les grèves 5 qui se déroulent entre 1975 et 1980 . Celles-ci surviennent dans les grands complexes industriels du secteur public. Selon les sociologues algériens ayant travaillé sur cette période, ces vagues de grève s'expliqueraient en premier lieu, par l'arrivée dans les entreprises d'Etat en expansion une nouvelle génération d'ouvriers adhérant largement au modèle de consommation occidentale et éprouvant moins de réticences que la génération issue de la guerre d'indépendance à faire grève [Chikhi, 1981, p. 61-62]. Ensuite, la seconde moitié des années 1970 serait caractérisée par un déplacement des conflits de l'université vers l'entreprise. En effet, certains militants de l'Union nationale des étudiants algériens réprimée et dissoute par les autorités s'intègrent pendant cette période dans le monde du travail. Ces derniers, souvent affiliés à des organisations politiques clandestines, pratiquent l'entrisme au sein de l'UGTA et deviennent ainsi les animateurs des grands mouvements de grève de cette période [Benamrouche, 1995, p. 96].

Face à cette stratégie d'entrisme et un mouvement social susceptible de leur échapper, les gouvernants cherchent au $5^{\mathrm{e}}$ congrès de l'UGTA en 1978 à circonscrire les conflits du travail en faisant en sorte qu'un ancien membre du parti communiste algérien, Demene Debih Abdallah, soit élu au poste de secrétaire général de la centrale syndicale. Mais de nouvelles vagues de grève en 1979 amènent les gouvernants à reprendre en main la direction de l'UGTA en faisant adopter par le congrès extraordinaire du FLN de juin 1980 un article modifiant les statuts du parti unique. Cet article 120 des statuts du FLN prévoit que «ne peut assumer des responsabilités au sein des organisations de masse que celui qui est militant structuré au sein du parti » [Taleb, 1985]. Il revient à écarter des postes à responsabilité des «organisations de masse »-par conséquent de l'UGTA - tous ceux qui ne seraient pas membres du FLN. Aussi, jusqu'à la fin des années 1980, la logique de l'UGTA est-elle restée la même : «subordonner le mouvement social aux fractions dirigeantes de l'État et non pas le faire animer et développer par des forces ouvrières autonomes » [Chikhi, 2001, p. 162-163].

4 Voir le site de l'UGTA : http://www.ugta.dz/pages.php?id=48, consulté le 10 mars 2006.

5 On notera que la grève est interdite dans le secteur public. Le soubassement idéologique de cette interdiction est simple : la grève est un moyen de défense des ouvriers contre les capitalistes exploiteurs, or l'employeur est l'État des travailleurs, par conséquent, elle n'a aucune raison d'être en régime socialiste. 


\section{Tunisie : une neutralisation progressive du champ syndical}

La Tunisie a été moins marquée par l'étatisation des moyens de production que l'Égypte - elle n'a jamais connu de rupture radicale avec les logiques du marché mais elle n'en a pas moins mis en place une formule corporatiste qui ressemble sur bien des points à celle de l'Égypte. Si dans la phase de lutte contre le colonisateur, le syndicat et les associations sportives et de jeunesse ont souvent constitué pour le leader $\mathrm{du}$ «mouvement national», Habib Bourguiba, un moyen de mobilisation politique les fédérations syndicales ont été subordonnées, une fois l'indépendance acquise. Prônant un «civisme contrôlé », le premier président de la République de Tunisie a tenté de mobiliser ses compatriotes au sein du parti bientôt unique (le NéoDestour) et des organisations, tout en les assujettissant au strict contrôle de l'État [Bellin, 1995, p. 126]. Ce faisant, les gouvernants ont instauré un corporatisme d'État dans lequel les salariés, à travers le syndicat unique, l'Union générale des travailleurs tunisiens (UGTT), les chefs d'entreprises, par le biais de l'Union tunisienne de l'industrie, du commerce et de l'artisanat (UTICA) et les paysans via l'Union nationale des agriculteurs tunisiens (UNAT) s'insèrent dans des organisations professionnelles uniques dont la mission est alors de soutenir les programmes présidentiels tout en étant un instrument de contrôle social [Redissi, 2004, p. 217]. Dans la formule corporatiste tunisienne, l'UGTT s'est imposée au milieu des années 1950 comme un partenaire à part entière au parti de Bourguiba à partir du moment où ce dernier s'est trouvé dans la nécessité de s'associer avec d'autres forces dans sa lutte contre la puissance coloniale française. Aux élections constituantes (1956) et législatives (1959) qui suivent, l'UGTT rejoint le Néo-Destour pour former avec l'UTICA et l'UNAT un «Front national» [Camau, 1983, p. 42]. Pour ne pas se faire déborder par une UGTT disposant de capacités de mobilisation non négligeables, le président Bourguiba met en place un dispositif visant à la neutraliser et à la subordonner au parti. Mais les techniques de corporatisation utilisées par le pouvoir bourguibien diffèrent de celles employées en Égypte, car il s'est agi ici d'affaiblir une organisation puissante ayant des « racines anciennes et profondes, antérieurs à la naissance du Néo-destour »[Camau, 1983, p. 42]. Divide ut imperes est au cœur de l'action du président Bouguiba à l'égard de l'UGTT: il suscite la création et la disparition d'une Union tunisienne du travail (UTT), puis coopte les représentants patentés de la centrale syndicale réunifiée «dans un dispositif général de pouvoir » [Camau, Geisser, 2003, p. 146-147]. La mise sous tutelle de l'UGTT est confirmée en mars 1963, lorsque le conseil national du Néo-Destour institutionnalise le parti unique et le contrôle direct sur les « organisations nationales » (UGTT, UTICA et UNAT).

Les régimes populistes autoritaires, en dépit de leur trajectoire historique différente, poursuivent des stratégies similaires à l'égard des centrales syndicales. Les salariés du secteur public bénéficient d'un certain nombre d'avantages matériels et symboliques et les cadres syndicaux, censés les représenter, sont cooptés par l'État dans une logique corporatiste autoritaire: ils sont présents dans les instances dirigeantes des entreprises, du parti gouvernemental, dans les parlements et parfois au sein même des gouvernements, mais ils doivent, en échange, filtrer les revendications salariales et sociales du salariat public pour les rendre compatibles avec les offres gouvernementales et rester crédibles auprès de leur base, ce qui parfois relève de la 
quadrature du cercle. Plus ou moins rapidement, selon les pays et souvent à l'aune de l'épuisement du modèle de développement promu par les régimes populistes autoritaires, les organisations syndicales ont eu tendance à perdre leur capacité de mobilisation au profit de leur fonction de contrôle social.

Les relations entre l'État et les organisations syndicales s'inscrivent dans un double mouvement d'étatisation de la société et de développement du clientélisme d'État. Le premier «se traduit par un élargissement maximum de l'appareil étatique (l'État-parti) sur les individus et les groupes » et «fait obstacle à l'émancipation des individus et à l'autonomisation des institutions sociales » tandis que le second répond «à la nécessité du maintien de l'ordre et à la distribution des ressources matérielles » [Camau, 1990, p.74].

Dans la Tunisie des années 1970, les relations entre l'UGTT et le pouvoir politique rendent compte des limites du clientélisme d'État tunisien, du caractère impensable d'une autonomisation syndicale à part entière et, partant, des difficultés rencontrées par la direction de l'UGTT à réguler les mouvements de protestations sociales. Contrairement aux fédérations syndicales égyptienne et syrienne qui sont restées complètement verrouillées, l'UGTT, d'instrument d'encadrement du pouvoir destourien, s'est transformée, le temps d'une courte décennie, en une structure d'opposition. En fait, il conviendrait plutôt de dire que, débordé par sa base, le leadership de l'UGTT s'est « laissé porter par la vague contestataire qui animait son organisation » [Camau, Geisser, 2003, p. 184].

\section{Autonomisation et politisation syndicales : l'exemple tunisien}

En Tunisie, cette transformation fait suite à la crise de 1969 qui a vu la remise en cause de la politique de collectivisation des terres décidé par le Premier ministre Ahmed Ben Salah et le rejet par les groupes sociaux les plus divers de l'emprise de l'État sur les différentes sphères sociales. Le gouvernement tunisien met alors en place les premiers linéaments d'une politique de libéralisation de l'économie qui débouche sur l'élargissement d'un secteur privé largement dépendant de l'État. Cette politique économique s'accompagne dans la première moitié des années 1970 d'une dynamisation de la croissance liée au développement d'une économie de rente. De surcroit, l'échec de la politique de collectivisation d'Ahmed Ben Salah a affaibli les capacités de mobilisation du parti-État, le Néo-Destour, et a fait de l'UGTT «un interlocuteur privilégié du pouvoir dans la mise en œuvre du compromis clientéliste » [Camau, 1989, p. 71].

Dans la première moitié des années 1970, la centrale bénéficie de l'embellie de la conjoncture économique. Sa direction négocie avec le gouvernement et le patronat de nombreux avantages pour les salariés (augmentation des salaires, conventions collectives, statuts protecteurs) et acquiert de la puissance grâce aux moyens financiers que lui procure la retenue à la source des cotisations.

La pouvoir bourguibien accepte, nolens volens, que l'UGTT occupe une position de partenaire privilégié, mais en échange sa direction se doit de filtrer les demandes de la base et d' «assurer leur compatibilité avec les offres gouvernementales » [Camau, Geisser, 2003, p. 183]. Or la centrale syndicale va, dans la première moitié des années 1970, progressivement rendre caduc ce compromis en polarisant les aspirations à 
l'autonomie de nouvelles couches de salariés mieux formées et parfois politisées au lycée ou à l'université. Certains éléments d'extrême gauche investissent les syndicats de service (notamment la banque), alors que le syndicat de l'enseignement supérieur, dominé par les communistes, fait son entrée en force au sein des organes dirigeants de la centrale [Toumi, 1989]. La direction syndicale se heurte ainsi à une contradiction, car elle se doit de répondre à des revendications de plus en plus radicales de la part de ses mandants tout en tentant de désamorcer les conflits sociaux [Khiari, 2003, p. 66]. La multiplication des grèves à la base pousse la direction de l'UGTT dans une logique contestataire. Le syndicat devient alors à la fin des années 1970 «le refuge et le fourrier des forces opposées au pouvoir» [Zghal, 1998, p. 10]. L'épreuve de force qui s'engage entre l'UGTT et le pouvoir bourguibien débouche le 26 janvier 1978 sur une grève générale, occasion d'émeutes et de manifestations, qui pousse Bourguiba à faire donner l'armée pour réprimer les manifestants. Mise au pas dans un premier temps, la centrale syndicale est rétablie dans son «autonomie » au congrès de Gafsa en 1981. Mais, affaiblie par une scission suscitée en son sein par le pouvoir (comme à la fin des années 1950), l'UGTT n'est en rien à l'origine des émeutes dites «de la faim » en 1984. Celles-ci sont révélatrices de l'épuisement d'un clientélisme d'État, fragilisé, cette fois-ci, par l'affaiblissement de ses capacités redistributives consécutif à la diminution des ressources rentières de la Tunisie du début des années 1980 [Camau, 1989].

Le président Ben Ali, arrivé aux affaires en 1987, intègre une UGTT moribonde dans un nouveau dispositif de pouvoir : elle cesse d'être liée organiquement au parti et à l'État ${ }^{6}$ et acquiert ainsi une sorte d'autonomie fonctionnelle qui la subordonne, mais d'une autre façon, au pouvoir politique. Bien que continuant à bénéficier du monopole de la représentation des salariés, sa direction, cooptée par la présidence de la République au Congrès de Sousse de 1989, est contrainte d'accepter les exigences du Programme d'ajustement structurel et de servir de relais du pouvoir. Le régime de Ben Ali utilise également le levier du financement de la centrale syndicale par l'État pour accentuer son emprise sur la direction de l'UGTT : il rend la centrale directement dépendante de subsides de l'État en abrogeant le système de retenue à la source des cotisations [Christopher A., 2002, p. 94]. Cette décision, combinée à la diminution du nombre d'adhérents fait de l'État le principal financier de la centrale. Certes, la direction l'UGTT dispose d'une marge de manœuvre importante sur le plan organisationnel, mais en contrepartie, elle se doit d'accepter la politique de libéralisation et de privatisation du régime et de se contenter de la critiquer à la marge.

En fait, l'ensemble des organisations syndicales du monde arabe est confronté, depuis la fin des années 1980, aux transformations socioéconomiques engendrées par les politiques de privatisation et de libéralisation consécutives à la mise en œuvre de l'ajustement structurel.

\section{Les syndicalismes arabes face à la libéralisation des économies}

L'association de l'autoritarisme et du libéralisme économique a des conséquences délétères tant sur les salariés que sur des mouvements syndicaux largement vidés de

6 Les cadres syndicaux n'occupent plus de fonctions politiques officielles et la centrale affirme sa neutralité à l'égard des partis reconnus. 
leur substance revendicative. D'une part, les politiques de privatisation et de libéralisation débouchent sur une contraction, tout au moins une stagnation du secteur public, qui regroupe le gros des troupes syndicales ; d'autre part, la précarisation, la flexibilité et le chômage peuvent à la fois être des facteurs de démobilisation de la population salariée et engendrer des mouvements incontrôlés de protestations sociales, hors du canal syndical officiel.

\section{L'Égypte : pérennité du corporatisme d'État}

En Égypte, la Confédération syndicale accepte, depuis le début des années 1990, la politique de libéralisation économique mise en œuvre par le pouvoir. En rupture avec son discours passé de refus de la privatisation, la CGSTE a accueilli favorablement, en 1991, la loi 203 qui transforme les entreprises publiques en sociétés par actions privatisables contrôlées par des holdings sectoriels. La direction syndicale a ainsi accepté de voir la représentation du personnel réduite au sein du conseil d'administration des entreprises soumises aux dispositions de la loi 203. Surtout, ce texte législatif permet d'exclure du système de représentation les sociétés dont au moins $50 \%$ du capital est privatisé car, si la législation du travail autorise l'élection d'un comité syndical dans les entreprises privées de plus de 250 employés, elle «n'impose la représentation d'élus des salariés au conseil d'administration de l'entreprise que dans le secteur public » [Ibrahim, 1998, p. 187] $]^{7}$.

Si le gouvernement a mis sept ans pour faire voter le nouveau code du travail dont la mise au point a été achevée en 1996, ce délai ne s'explique pas par la capacité d'obstruction de la CGSTE, mais plutôt par la crainte du pouvoir politique d'avoir à faire face à des mouvements sociaux spontanés et incontrôlés. Il met en évidence les limites inhérentes à la neutralisation du champ syndical. En effet, la centrale syndicale se retrouve dans l'incapacité d'avoir prise sur les salariés qu'elle est censée représenter. Elle ne peut ni remplir la fonction de contrôle social que le pouvoir lui a prescrite, ni contrôler les mouvements de contestation au niveau des entreprises, ce qui pousse les gouvernants à utiliser l'appareil répressif à l'égard des grévistes ou des manifestants ${ }^{8}$.

\section{La Tunisie : un néo-corporatisme en trompe l'oeil}

En Tunisie, la formule de contrôle des demandes de la base dans un contexte de libéralisation de l'économie rencontre également ses limites, bien qu'elle soit plus souple qu'en Égypte.

Le système de régulation des revendications que met en place le régime de Ben Ali au début des années 1990 s'inscrit dans le cadre d'arrangements corporatistes impliquant l'État, l'organisation patronale (UTICA) et l'UGTT. Il définit une nouvelle politique de négociations qui remplace les discussions annuelles sur les salaires par un accord de trois ans prévoyant l'échelonnement des augmentations salariales en vue

${ }^{7}$ L'affiliation syndicale du salarié du privé n'est pas un droit mais une simple possibilité. Elle est limitée aux entreprises de plus de 250 salariés et soumise à l'accord de l'employeur. Voir Françoise Clément, [1998, p. 44].

8 Pour le détail des mouvements de grève et leur répression, voir Posusney [1997, p. 230-243] 
d'en modérer l'ampleur - pendant son application, les revendications salariales sont gelées - [Hamzaoui, 2002, p. 373]. Cette politique de «concertation» entre les «partenaires sociaux » pourrait suggérer le passage d'un corporatisme d'État à un néocorporatisme. En effet, les directions de l'UGTT et l'UTICA semblent être intégrées dans un processus d'élaboration tripartite (syndicat, patronat et État) de la politique salariale par l'institutionnalisation de leur rôle et la pratique systématique du compromis [Alexander, 1996, p. 271].

Cependant, ce néo-corporatisme est largement en trompe-l'œil : en effet, dans le cadre de ces arrangements corporatistes des années 1990, les fonctions de contrôle et de discipline du leadership sur les militants syndicaux sont hypertrophiées au détriment de la contrepartie supposée du néo-corporatisme, i.e. la participation active de la direction syndicale à «la définition, voire à la mise en œuvre des politiques publiques [1a] concernant» [Jobert, Muller, 1987, p. 160]. Par conséquent, le leadership de l'UGTT se retrouve en porte-à-faux vis-à-vis du monde salarié : il est souvent débordé par l'action des syndicats de base au niveau de l'entreprise.

En dépit du contrôle sécuritaire, certaines fédérations régionales continuent de disposer «d'une potentialité de mobilisation autonome» [Geisser, Gobe, 2005, p. 306]. Elles se sont saisies à plusieurs reprises depuis 2001 de la situation régionale au Moyen-Orient (question palestinienne et invasion américaine de l'Irak) pour organiser des manifestations.

Aussi l'UGTT ne peut-elle être assimilé à une simple courroie de transmission du pouvoir politique. Elle est plutôt « le maillon faible des agences de pouvoir » [Camau, Geisser, 2003] . La centrale syndicale a montré une autre fois en 2005 des velléités d'autonomie : la direction de l'UGTT a refusé à une courte majorité de participer à l'élection de la seconde chambre du Parlement récemment créée, la Chambre de conseillers, - censée représenter les acteurs de la société civile - parce que le pouvoir voulait directement interférer dans le choix de « ses » candidats [Geisser, Gobe, 2007].

Autrement dit, la politique de cooptation de la direction syndicale par le président Ben Ali, bien que cela soit l'un de ses objectifs principaux, ne peut empêcher ni l'affirmation de dissensions au sein de l'appareil syndical [Khiari, 2003, p. 184], ni le développement de contestations sociales. Depuis 2000, de nombreuses protestations ouvrières se sont exprimés en partie en dehors d'un mouvement syndical refusant de reconnaître le caractère légal des grèves ou intervenant a posteriori dans les conflits sociaux. En raison de son «ancrage dans les relations économiques et sociales » et de la nécessité de tenir compte des revendications de sa base, «la centrale ne peut être parfaitement disciplinée » [Hibou, 2006, p. 150].

\section{L'Algérie : entre logique corporatiste autoritaire et pluralisme syndical}

Cette forme de néo-corporatisme en trompe l'œil concerne également l'Algérie. Le bref moment de libéralisation politique qui suit les émeutes de 1988 débouche en 1990 sur la reconnaissance du pluralisme syndical par la législation algérienne et la reconnaissance du droit de grève dans le secteur public. Toutefois, les autorités algérienne ne vont pas jusqu'au bout de la logique impliquée par la proclamation du pluralisme. Elles refusent de légaliser le syndicat islamique, organisation capable de mobiliser les salariés en faveur du Front islamique du salut (FIS), le principal parti 
islamiste algérien. Certes, l'UGTA est devenue en 1990 indépendante du FLN, mais elle est intégrée par le pouvoir militaire en 1991 dans deux structures de concertation, une bipartite (gouvernement, représentants des salariés) et l'autre tripartite (gouvernement, représentants des employeurs et des salariés). Ce cadre de négociation tripartite est utilisé par les autorités «comme preuve d'une acceptation sociale des mesures [qu'il a] dû prendre dans le cadre du Plan d'ajustement structurel » [Benamrouche, 2000, p. 253].

La centrale syndicale demeure ainsi attachée à ses relations privilégiées avec le pouvoir qui permettent aux responsables syndicaux d'être partie prenante, même de manière marginale aux prises de décision en matière économique. En échange de ces avantages, la direction de l'UGTA participe activement à la mise en place du Comité national de sauvegarde de l'Algérie qui, créé le 31 décembre 1991, a pour mission de préparer l'opinion à l'annulation du second tour des élections législatives de janvier 1992 qui auraient permis aux FIS d'accéder légalement au pouvoir [Benamrouche, 1995, p. 53]. L'UGTA reste, au cours de ces quinze dernières années, fidèle à son rôle de courroie de transmission du pouvoir: elle a appelé à soutenir les candidats du pouvoir militaire (Liamine Zeroual en 1995, Abdelaziz Bouteflika en 1999 et 2004) et n'a de cesse de faire campagne pour une «participation massive» aux différentes élections ${ }^{9}$. Dans le champ économique, la centrale n'a jamais défendu publiquement le dossier d'une entreprise en difficulté. L'UGTA, hier comme aujourd'hui, se doit de jouer «le rôle d'instrument d'une politique d'austérité qu'au nom des impératifs du développement, il est chargé de faire accepter par les salariés » [Weiss, 1970, p. 60].

$\mathrm{Si}$ la puissance publique a reconnu 53 organisations professionnelles, elles refusent de les considérer comme des partenaires sociaux et de les intégrer dans des dispositifs de concertation, y compris au sein des conseils d'administration des divers organismes sociaux. Ces syndicats, essentiellement à base professionnel sectoriel, se concentrent principalement dans la fonction publique ${ }^{10}$ et sont l'objet d'un harcèlement permanent de la part des autorités. Ces dernières recourent de manière systématique à la justice contre les grévistes et ont restreint l'exercice du droit de grève en 2004 en assimilant «toute grève dite illimitée à un abandon de poste » [Bennadji, 2006, p. 194].

Dans le contexte actuel, l'UGTA ne risque pas de perdre son statut de partenaire privilégié du pouvoir. En ignorant et en réprimant les syndicats « autonomes », il s'agit pour les gouvernants d'éviter l'émergence d'une élite sociale susceptible de contester celle qui dépend du pouvoir politique, en l'occurrence la direction de la centrale syndicale algérienne.

\footnotetext{
9 Abdenasser Djabi, Le syndicalisme entre surpolitisation et désir d'autonomie, http://www.algeriainterface.com, consulté le 10 mars 2006.

10 On citera parmi les syndicats «autonomes » les plus importants le SNAPAP (Syndicat national autonome du personnel de l'administration publique); SNPSP (Syndicat des praticiens de santé publique); SNPSSP (Syndicat des praticiens spécialistes de santé publique); SNMASM (Syndicat national des maîtres assistants en sciences médicales); SPLA (Syndicat des pilotes de ligne d'Air Algérie); SNTMA, (Syndicat national des techniciens de la maintenance aérienne), SNPCA (Syndicat national du personnel du contrôle aérien), SNOMMAR (Syndicat national des officiers de la marine marchande), SATEF (Syndicat autonome des travailleurs de l'éducation et de la formation), CLA (Conseil des lycées d'Alger) ; CNAPEST (Conseil national autonome des professeurs de l'enseignement secondaire et technique); CNES (Conseil national des enseignants du supérieur).
} 


\section{Les syndicats dans le monde arabe : courroies de transmission des régimes autoritaires?}

L'évolution récente de l'histoire des fédérations syndicales confirmerait les hypothèses d'Emma C. Murphy [1999, p. 37-39] sur les mutations récentes du corporatisme autoritaire. Selon la politologue britannique, les conséquences sociopolitiques de la libéralisation économique et de l'apparente «démocratisation » qu'ont pu connaître certains pays arabe à la fin des années 1980 déboucheraient sur une «restructuration tactique des mécanismes de contrôle» et une «forme désarticulée » de corporatisme d'État. Sous l'impact des dynamiques sociales induites par les transformations économiques, les groupes sociaux que sont censées représenter les « organisations fonctionnelles de médiation » se diversifient et ne peuvent plus se reconnaître dans des institutions corporatistes dont l'assise sociale se réduit comme une peau de chagrin. Aussi, ces dernières ont-elles tendance à tourner à vide et à ne plus avoir de prise sur la réalité sociale, se contentant d'apporter, à intervalle régulier, leur soutien aux gouvernants. Dans le même temps, ceux-ci continuent de voir les syndicats comme des institutions qu'il convient de mettre sous tutelle pour empêcher leur éventuelle autonomisation. En ce sens, la période de politisation qu'a connue l'UGTT fait figure d'exception dans le paysage syndical tant maghrébin que moyenoriental $^{11}$.

Du corporatisme d'État des années 1960 et 1970, il ne reste plus que la dimension répressive. Certes, il existe des différences entre la Fédération tunisienne et ses homologues égyptien et algérien complètement subordonnées au pouvoir politique quasiment sans interruption depuis les années 1960. L'Égypte, constitue un exemple extrême de subordination d'un syndicat centralisé n'ayant aucune prise sur les mouvements sociaux [Longuenesse, 1998]. Mais par delà les divergences des trajectoires historiques des différents pays, les centrales syndicales ne semblent fonctionner que pour empêcher l'émergence d'une capacité revendicative susceptible de remettre en cause non seulement une politique économique, mais aussi des privilèges et des pratiques de corruption.

In fine, dans les États autoritaires de manière générale, les Fédération syndicales constituent des rouages - certes pas toujours entièrement soumis -, qui participent au maintien de l'ordre public et de la paix sociale.

\section{Bibliographie}

AHDJouj A. (1992), Algérie, État, pouvoir et société (1962-1965), Éditions Épigraphe, Alger.

AlEXANDER C. (1996), Between Accomodation and Confrontation : State, Labor and Development in Algeria and Tunisia, PH. D., Department of Political Science, Duke University.

11 À cet égard, la politisation et le pluralisme du syndicalisme marocain en font un cas à part dans le paysage syndical arabe. Voir Myriam Catusse [2001]. 
AYUBI N. (1995), Over-stating the Arab State. Politics and Society in the Middle East, I.B. Tauris, Londres et New York, 1995.

BADIE B., Hermet G. (2001), La politique comparée, Paris, Armand Colin.

Bellin E., «Civil Society in Formation : Tunisia », dans NorTON A. R., Civil Society in the Middle East, Leiden, New York et Cologne, E.J. Brill, 1995, p. 120-147.

BENAMROUCHE A. (1995), «État, conflits sociaux et mouvement syndical en Algérie (1962-1995) », Monde arabe Maghreb-Machrek, n¹48, avril-juin, p. 43-54.

BenAmRouche A. (2000), Grèves et conflits politiques en Algérie, KarthalaInstitut Maghreb-Europe, Paris, 2000.

BENNADJi C. (2006), «Algérie : la fin de la crise politique ?», L'Année du Maghreb 2004, CNRS Éditions, Paris, p. 175-206.

BIANCHI R. (1989), Unruly Corporatism. Associational life in the twentiethcentury Egypt, Oxford University Press, Oxford et New York, 1989.

CAMAU M. (1989), La Tunisie, PUF, QSJ, Paris, 1989.

CAMAU M. (1990), «État, espace public et développement. Le cas tunisien », dans El-Malki H., SAnTuCCi J.-C. (dir.), État et développement dans le Monde arabe. Crises et mutations au Maghreb, Éditions du CNRS, Paris, p. 67-78.

CAmau M., GeISSER V. (2003), Le syndrome autoritaire. Politique en Tunisie de Bourguiba à Ben Ali, Presses de Sciences Po, Paris.

CATUSSE M. (2001), «La métamorphose de la question syndicale au Maroc », Critique économique, 5, printemps, p. 39-67.

CHIKHI S. (1982), «La classe ouvrière aujourd'hui en Algérie », Les Temps Modernes, juillet-août, p. 57-79.

CHIKHI S. (2001), « Syndicat et classe ouvrière », dans DJERBAL D., BENGUERNA M., Mouvement social \& modernité. Hommage à Saïd Chikhi, Naqd/SARP, p. 157169.

Christopher A. (2002), «Enterprise Reform and labor in North Africa », in Cook Linda J. et Posusney M. P. (dir.), Privatization and Labor: Responses and Consequences in Global Perspective, Edward Elgard Publishing, Cheltenham et Northampton, p. 83-110.

Clement F. (1998), «Libéralisation du rapport salarial en Égypte », Monde Arabe Maghreb-Machrek, n 162, octobre-décembre, p. 39-56. 
DROZ-VINCENT P. (2004), Moyen-Orient: pouvoirs autoritaires, sociétés bloquées, PUF, Paris.

FAHMY N. S. (2002), The politics of Egypt. State-Society Relationship, RoutledgeCurzon, Londres et New-York.

GALlissot R. (1987), Maghreb-Algérie. Classe et nation, Arcantère Éditions, tome I, Paris.

GEISSER V. et Gobe E. (2007), «Des fissures dans la maison Tunisie ? Le régime de Ben Ali face aux mobilisations protestataires », L’Année du Maghreb édition 2005-2006, CNRS Éditions, Paris.

GeISSER V. et GOBE E., (2005), «Le président Ben Ali entre les jeux de coteries et l'échéance présidentiel de 2004 », Annuaire de l'Afrique du Nord 2003, CNRS Éditions, Paris.

GoBE E., (2006), « Corporatisme, syndicalisme et dépolitisation », dans PICARD E. (dir.), La politique dans le monde arabe, Armand Colin, coll. «U », Paris, p. 171192.

HamzaOui S. (2002), «Champ politique et syndicalisme en Tunisie », Annuaire de l'Afrique du Nord 1999, CNRS Éditions, Paris, p. 369-380.

Heydemann S. (1999), Authoritarianism in Syria-Institutions and Social Conflict 1946-1970, Cornell University Press, Ithaca.

HibOU B. (2006), La force de l'obéissance : économie politique de la répression en Tunisie, La Découverte, Paris.

IBRAHIM G. (1998), «Représentation syndicale et transition libérale en Égypte. Lecture des élections de 1996 », Égypte/Monde arabe, $\mathrm{n}^{\circ} 33,1^{\text {er }}$ sem., p. 181-217.

Jobert B., Muller P., (1987), L'État en action: politiques publiques et corporatismes, PUF, Paris.

KHIARI S. (2003), Tunisie, le délitement de la cité. Coercition, consentement, résistance, Karthala, Paris.

KIEnle E. (2000), A Grand Delusion. Democracy and Economic Reform in Egypt, Londres et New York, I.B. Tauris.

LINZ J. J. (2006), Régimes totalitaires et autoritaires, Paris, Armand Colin.

LONGUENESSE E. (1999), «Maroc, Tunisie, Égypte : transition libérale et 
recompositions syndicales », Monde arabe Maghreb-Machrek, $\mathrm{n}^{\circ} 162$, octobredécembre, p. 3-5.

Longuenesse E. (2007), Professions et société au Proche-Orient. Déclin des élites, crise des classes moyennes, Rennes, PUR.

Murphy E. C. (1999), Economic and Political Change in Tunisia: From Bourguiba to Ben Ali, St Martin's Press, New York - MacMillan Press, Londres.

PICARD E. (dir.) (2006), La politique dans le monde arabe, Armand Colin, coll. « $\mathrm{U} »$, Paris.

POSUSNEY M. P. (1997), Labor and the State in Egypt. Workers and Economic Restructuring, Columbia University Press, New York.

Posusney M. P. (1992), «Labor as an Obstacle to Privatization: The Case of Egypt », dans HARIK I., SUllivan D. J. (dir.), Privatization and Liberalization in the Middle East, Indiana University Press, Bloomington et Indianapolis, p. 81-105.

REDISSI H. (2004), «L'autoritarisme de Bourguiba: continuités et ruptures », dans CAMAu M., GeISSER V. (dir.), Habib bourguiba. La trace et l'héritage, Karthala, Coll. Science politique comparative, Paris et Aix-en-Provence, p 209-222.

Richards A., Waterbury J. (1990), A Political Economy of the Middle East. State, Class and Economic Development, Westview Press, Boulder San Francisco, Oxford.

SCHMITTER P. (1974), «Still the Century of Corporatism? », The Review of Politics, vol. 36, n ${ }^{\circ}$, p. 85-131.

TALEB A. (1985), «Les rapports parti-syndicat en Algérie à travers l'application de l'article 120 des statuts du FLN», dans SRAÏEB N. (dir.), Le mouvement ouvrier maghrébin, Éditions du CNRS, Paris, p. 183-196.

Toumi M. (1989), La Tunisie de Bourguiba à Ben Ali, PUF, Coll. Politique d'aujourd'hui, Paris.

WeISS F. (1970), Doctrine et action syndicale en Algérie, Éditions Cujas, Paris.

ZGHAL R. (1998), «Nouvelles orientations du syndicalisme tunisien », Monde Arabe Maghreb-Machrek, $\mathrm{n}^{\circ}$ 162, octobre-décembre, p. 6-17. 